\title{
Fatty Acid Desaturases in the Chloroplast and Endoplasmic Reticulum Promote Susceptibility to the Green Peach Aphid Myzus persicae in Arabidopsis thaliana
}

\author{
Jiamei Li, Aravind Galla, Carlos A. Avila, Kaitlin Flattmann, Kaleb Vaughn, and Fiona L. Goggin ${ }^{\dagger}$ \\ Department of Entomology and Plant Pathology, University of Arkansas, Fayetteville, AR 72701, U.S.A.
}

Accepted 25 January 2021.

Fatty acid desaturases (FADs) in plants influence levels of susceptibility to multiple stresses, including insect infestations. In this study, populations of the green peach aphid (Myzus persicae) on Arabidopsis thaliana were reduced by mutations in three desaturases: AtFAB2/SSI2, which encodes a chloroplastic stearoyl-[acyl-carrier-protein] 9-desaturase, and $A t F A D 7$ or AtFAD3, which encode $\omega$-3 FADs in the chloroplast and endoplasmic reticulum (ER), respectively. These data indicate that certain FADs promote susceptibility to aphids and that aphids are impacted by desaturases in both the chloroplast and ER. Aphid resistance in ssi2, fad3, and fad7, singly or in combination, might involve altered signaling between these subcellular compartments. C18:1 levels are depleted in ssi2, whereas C18:2 accumulation is enhanced in $f a d 3$ and $f a d 7$. In contrast, fad 8 has higher than normal C18:2 levels but also high C18:1 and low C18:0 and does not impact aphid numbers. Potentially, aphids may be influenced by the balance of multiple fatty acids (FAs) rather than by a single species, with C18:2 promoting aphid resistance and C18:1 promoting susceptibility. Although the fad7 mutant also accumulates higher-than-normal levels of C16:2, this FA does not contribute to aphid resistance because a triple

Current address for Carlos A. Avila: Department of Horticultural Sciences, Texas A\&M AgriLife Research and Extension Center, Weslaco, TX 78596, U.S.A.

Current address for Kaitlin Flattmann: University of Tennessee Health Science Center, Memphis, TN 38163, U.S.A.

Current address for Kaleb Vaughn: Department of Medicine-Pediatrics, University of Oklahoma School of Community Medicine, Tulsa, OK 74135, U.S.A

${ }^{\dagger}$ Corresponding author: F. L. Goggin; fgoggin@uark.edu

Funding: This work was supported by the Agricultural and Food Research Initiative Foundational Program of the United States Department of Agriculture National Institute of Food and Agriculture (grant number 2015-67013-23412 to F. L. Goggin), the National Science Foundation Division of Integrative Organismal Systems (grant number IOS 0951287 to F. L. Goggin), the University of Arkansas Chancellor's Innovation Fund, and the Arkansas Agricultural Experiment Station.

*The $\boldsymbol{e}$-Xtra logo stands for "electronic extra" and indicates there is supplementary material published online.

The author(s) declare no conflict of interest. mutant line that lacks detectable levels of C16:2 (fad2fad6$\mathrm{fad} 7$ ) retains comparable levels of aphid resistance as $\mathrm{fad} 7$. In addition, aphid numbers are unaffected by the fad5 mutation that inhibits C16:1 synthesis. Together, these results demonstrate that certain FADs are important susceptibility factors in plant-aphid interactions and that aphid resistance is more strongly associated with differences in $\mathrm{C18}$ abundance than C16 abundance.

Keywords: FAD2, FAD3, FAD6, FAD7, SSI2, FAB2, fatty acid desaturase, GLABRA1, green peach aphid, stearoyl-acyl carrier protein desaturase

Aphids, a large group of piercing-sucking insects specialized to feed on phloem sap, are among the world's most common and damaging insect pests. There are more than 4,300 aphid species, and nearly all field crops, horticultural plants, and forest trees are impacted by one or more species (Blackman and Eastop 1994, 2000). Aphid attack can cause significant direct damage to their hosts, including chlorosis, wilting, and stunted growth (Goggin et al. 2017). Furthermore, aphids can also cause extensive indirect damage by transmitting viruses and promoting the growth of sooty mold (Minks and Harrewijn 1989; Stevens and Lacomme 2017). One of the most cosmopolitan aphid pests, the green peach aphid (Myzus persicae), has a worldwide distribution and feeds on more than 50 plant families, including a diversity of crops, such as potato, tobacco, sugarbeet, cabbage, peach, and spinach (Baker 1994; Blackman and Eastop 2000). This damaging species can transmit over 100 plant viruses, including potato leaf roll virus, cauliflower mosaic virus, turnip mosaic virus, pea leaf roll virus, radish yellows virus, and cucumber mosaic virus (Kennedy et al. 1962; Kuhar et al. 2009). Further complicating their management, the green peach aphid and certain other aphids have also developed resistance to multiple insecticides (Bass et al. 2014; Foster et al. 2017). The interaction of the green peach aphid with the model plant Arabidopsis thaliana represents a useful experimental system in which to explore plant-aphid interactions (Louis and Shah 2013).

Because of the importance of aphids as crop pests and the prevalence of pesticide resistance among these pests, host plant resistance is a particularly important component of aphid management (Van Emden 2017). However, our ability to select for aphid resistance in crops is impeded by our limited understanding of the host factors that influence aphids. These so-called "stealthy" herbivores can evade many of the plant defenses normally triggered by insect damage, although they induce a variety of reactions that overlap with plant defenses against pathogens (Kaloshian and Walling 2005; Walling 2008). In addition to constitutive and 
induced defenses, the success of invaders such as pathogens or aphids is also influenced by so-called susceptibility factors or host traits that can promote the infestation process (Eckardt 2002; Li et al. 2020; van Schie and Takken 2014). Because of their key role in modulating immunity, susceptibility factors are common targets for genome editing and other approaches to engineer enhanced disease resistance in crops (Zaidi et al. 2018).

Many susceptibility factors are negative regulators of plant immunity that most likely function to manage the balance between growth and defense in the plant. Without proper regulation by other genes, receptors for pathogen detection or immune signaling and defense genes often cause excessive cell death and stunted growth in plants (Ning et al. 2017). In the absence of infection, negative regulators limit these costs to plant growth by suppressing expression of immune genes or mediating degradation of their protein products; however, in the presence of a virulent pathogen that is not effectively recognized by the host plant, these negative regulators can also function as susceptibility factors, impeding appropriate immune responses $(\mathrm{Li}$ et al. 2020; van Schie and Takken 2014). For example, NRL1 in potato suppresses cell death by promoting proteolysis of a positive regulator of immunity (SWAP70), and it promotes infection by virulent strains of the late blight pathogen Phytopthora infestans that express the Pi02860 effector protein, because Pi02860 causes NRL1 to degrade SWAP70 even in the presence of the pathogen (He et al. 2018). Another example of a susceptibility factor is the SSI2 fatty acid desaturase (FAD) in rice that promotes infection by the causal agents of leaf blight and blast (Jiang et al. 2009; Li et al. 2020). Interestingly, loss of function of SSI2 in Arabidopsis reduces susceptibility to the green peach aphid Myzus persicae (Louis et al. 2010a; Pegadaraju et al. 2007), and FATTY ACID DESATURASE 7 (FAD7) also promotes susceptibility to aphids in Arabidopsis and tomato (Avila et al. 2012). Thus, FADs may represent important susceptibility factors for aphid infestation and further research is warranted to understand their impacts on plant-aphid interactions.

Fatty acid desaturases (FADs) located in the plastids or the endoplasmic reticulum (ER) introduce double bonds into the acyl chain of fatty acids (FA), regulating the level of desaturation of FAs incorporated into membrane- or storage lipids (Fig. 1). The majority of plant FAs have acyl chains of 18 or 16 carbons (C18 or C16); they are synthesized in plastids and either incorporated into galactoglycerolipids or exported to the ER for phospholipid synthesis. FA desaturation is initiated in the chloroplast by FAD5, which acts on C16:0, or by S-ACPDES7 (stearoyl-[acyl-carrier-protein] 9-desaturase 7), which

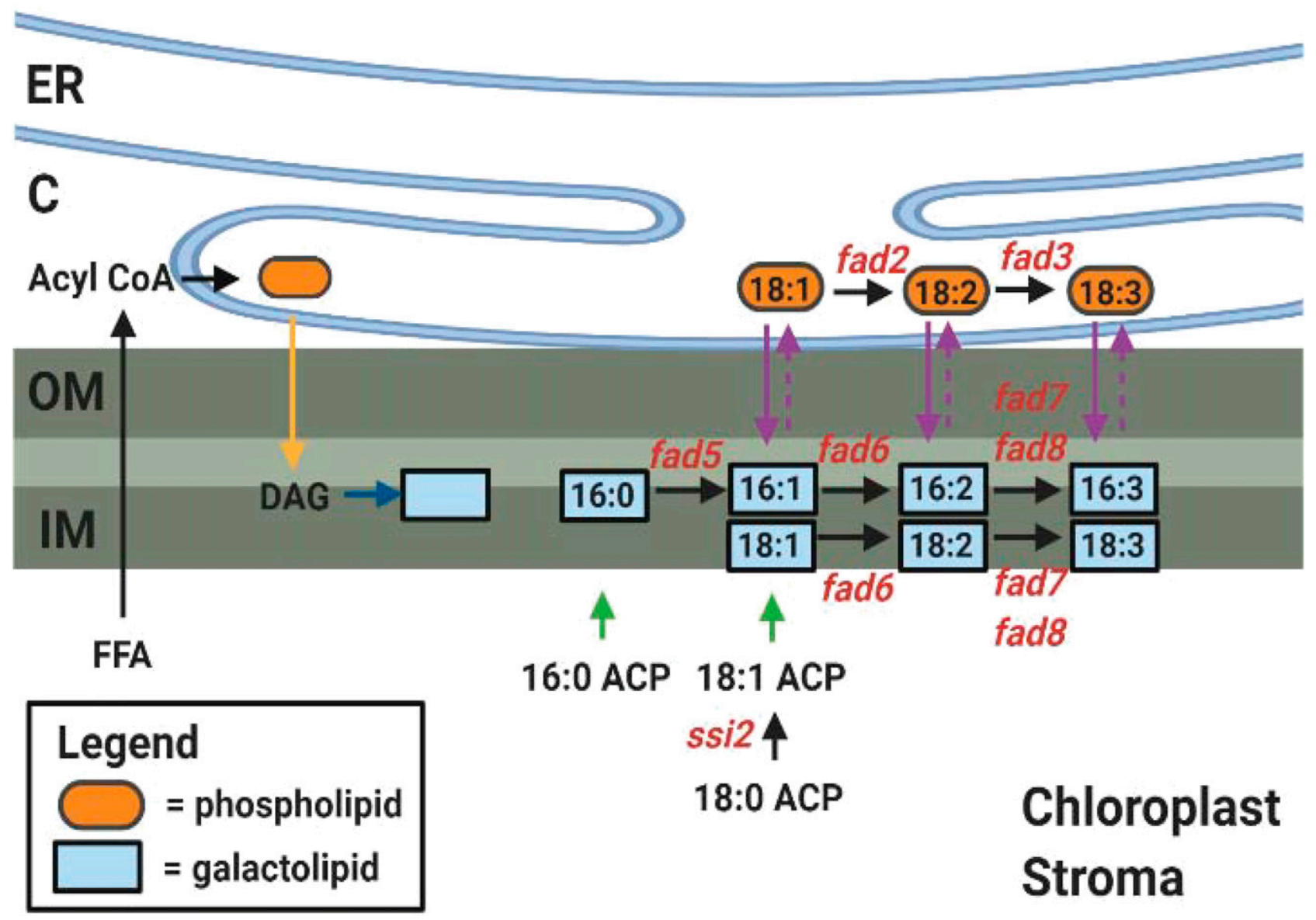

Fig. 1. Fatty acid metabolism in the chloroplast and endoplasmic reticulum (ER). Mutations that inhibit FA desaturation are represented in red. C18:0 bound to an acyl carrier protein (18:0 ACP) is desaturated in the chloroplast stroma by a stearoyl-acyl carrier protein desaturase encoded by FAB2/SSI2. All other desaturases shown here are membrane-associated proteins. Esterified C16:0, C16:1, and C16:2 are desaturated exclusively in the chloroplast, whereas esterified C18:1 and C18:2 can be desaturated in the chloroplast or ER. Extensive metabolic interconnections exist between these two subcellular compartments. FAs synthesized in the chloroplast are either incorporated into galactolipids via the prokaryotic pathway (green arrows) or are exported in the form of free FAs to the ER, in which they are used for phospholipid synthesis. Phospholipids synthesized in the ER are also a source of diacylglycerol for production of chloroplast galactolipids via the eukaryotic pathway (blue arrow). It is currently uncertain which phospholipid or phospholipid derivative is transported from the ER to the chloroplast for this purpose (yellow arrow). Lipids can also be exchanged from the ER to the chloroplast via the trigalactosyldiacylglycerol transport complex (solid purple arrows) or from the chloroplast to the ER via as-yet-uncharacterized mechanisms (dashed purple arrows). $\mathrm{C}=$ cytosol, $\mathrm{IM}=$ inner membrane of chloroplast, $\mathrm{OM}=$ outer membrane of chloroplast. This figure was created with BioRender and represents a synthesis of models presented by Karki et al. (2019), Mehrshahi et al. (2014), and Somerville and Browse (1996). 
acts on C18:0 and is encoded by the FATTY ACID BIOSYNTHESIS 2 gene (FAB2, also known as SSI2) (Kachroo et al. 2005; Kunst et al. 1989). The resulting monoenoic FAs can be further desaturated by chloroplast-localized FAD6, which acts on both $\mathrm{C} 18: 1$ and $\mathrm{C} 16: 1$, or by ER-localized FAD2, which desaturates C18:1 (Browse et al. 1989; Lemieux et al. 1990). In addition, there are three $\omega-3$ FADs that convert dienoic FAs to trienoic FAs (Arondel et al. 1992; Gibson et al. 1994): FAD3, which is localized in the ER and synthesizes C18:3, and FAD7 and FAD8, which are located in the chloroplast and generate both $\mathrm{C} 18: 3$ and C16:3 (Browse et al. 1986, 1993; Dyer and Mullen 2001; Froehlich et al. 2003; McConn et al. 1994). Trienoic FAs are the most abundant polyunsaturated FA species in plant membrane lipids and make up about 70 to $80 \%$ of the FAs in the chloroplast membrane lipids (Harwood 1980; Yaeno et al. 2004).

FADs can modulate levels of plant defenses to many stresses, including pathogens and insects (Upchurch 2008). Polyunsaturated FAs generated by FADs are precursors for multiple oxylipins that contribute to plant defense. C18:3 is required for synthesis of 12-oxo-phytodienoic acid, jasmonic acid, and other jasmonates, and so $\omega-3$ FADs are critical to jasmonate-dependent defenses against many pests; as a result, the fad3fad7fad 8 triple mutant in Arabidopsis is highly susceptible to fungus gnat larvae as well as oomycete infection (McConn et al. 1997; Vijayan et al. 1998). In addition, dienoic and trienoic FAs give rise to a multitude of other oxylipins via lipoxygenases, hydroperoxide lyase, and other enzymatic and nonenzymatic pathways, and many of these oxylipins function as phytoalexins or defense signals (Blée 2002). For example, green leaf volatiles generated by the hydroperoxide lyase pathway attract natural enemies, prime defense signaling in neighboring foliage, and can be directly toxic to insects and pathogens (Scala et al. 2013). In addition to providing substrates for oxylipin synthesis, FADs also have indirect effects on defense signaling; for example, suppression of the Arabidopsis FAD7 and $F A D 8$ genes reduces the accumulation of reactive oxygen intermediates and decreases resistance to bacterial pathogen Pseudomonas syringae (Yaeno et al. 2004).

While FADs contribute to plant defenses against certain pests, they appear to increase susceptibility to other stressors. Suppression of $O s F A D 7$ and $O s F A D 8$ expression in rice confers resistance to the rice blast fungus Magnaporthe grisea (Yara et al. 2007, 2008), and loss of function of FAD7 in tomato and Arabidopsis also confers aphid resistance (Avila et al. 2012). Settling, survival, and fecundity of the potato aphid (Macrosiphum euphorbiae) is significantly lower on tomato mutants with impaired FAD7 function compared with wild-type (WT) controls (Avila et al. 2012). Furthermore, the ssi2 mutation in FAB2, which impairs function of S-ACP-DES7, decreases population growth of the green peach aphid on Arabidopsis (Pegadaraju et al. 2007). Thus, FADs may act as susceptibility factors in certain biotic interactions, including aphid infestation. The goals of this study were to characterize the effects of the major FADs in Arabidopsis on susceptibility to the green peach aphid and to correlate these effects with foliar FA profiles.

\section{RESULTS}

Influence of FATTY ACID BIOSYNTHESIS 2 (FAB2) and $F A D 5$ on aphid resistance and $F A$ profiles.

To assess the effects of desaturases that synthesize monoenoic FAs on aphids, we assayed FA profiles and aphid population growth on the Arabidopsis ssi 2 and fad5 mutants, which inhibit the synthesis of $\mathrm{C} 18: 1$ and $\mathrm{C} 16: 1$, respectively. The ssi2 mutation in the $F A B 2$ gene causes a $10-20$ fold decrease in the $\Delta 9$ desaturase activity of S-ACP-DES7, which converts C18:0 to C18:1 in the chloroplast stroma (Fig. 1) (Kachroo et al. 2001). The ssi2 mutant was stunted (Supplementary Fig. S4) and supported significantly fewer aphids than the WT background for this mutant, the Nossen ecotype (Fig. 2). The lower aphid numbers on ssi2 appeared to be due to reduced adult fecundity rather than to increased mortality, because dead aphids were absent in most cages. The reduced aphid fecundity observed on ssi 2 is consistent with previous reports that this mutant has antibiotic effects on the green peach aphid (Louis et al. 2010a and b; Pegadaraju et al. 2005). Compared with Nossen, ssi 2 had more than 16-fold higher levels of C18:0 accumulation and significantly lower levels of C18:1, C18:2, and C18:3 but did not promote C16:0 accumulation or deplete C16:1 levels (Fig. 3). This is consistent with a previous report that both the mutant and WT isomers of S-ACP-DES7 have a very strong substrate preference (88:1) for C18:0 rather than C16:0 (Kachroo et al. 2001). The stunted growth of ssi2 (Supplementary Fig. S4) could potentially reduce host suitability for aphids; however, prior studies have shown that petiole exudates from ssi2 inhibit aphid growth on artificial diets, indicating that aphid resistance in $s s i 2$ is due, at least in part, to altered plant chemistry rather than to altered plant morphology (Louis et al. 2010a). In contrast to ssi2, the fad5 mutation did not significantly impact aphid population growth (Fig. 2). This mutation impairs a chloroplastic palmitoyl-monogalactosyldiacylglycerol $\Delta 7$-desaturase that coverts $\mathrm{C} 16: 0$ to $\mathrm{C} 16: 1$ (Fig. 1) (Heilmann et al. 2004). Compared with WT plants, the fad5 mutant had similar C18 FA levels, significantly higher C16:0 levels, and significantly lower levels of C16:3 accumulation (Fig. 3). The differences in aphid susceptibility and FA profiles between ssi2 and fad5 suggest that $\mathrm{C} 18$ FAs have more influence on aphid numbers than C16 FAs.

\section{Influence of $F A D 2$ and $F A D 6$ on aphid resistance and $\mathrm{FA}$ profiles.}

To assess the effects of desaturases that synthesize dienoic FAs on aphids, aphid population growth and foliar FA profiles were also measured in mutants with impairments in $\Delta-12$ FADs in the ER $(f a d 2)$ or chloroplast $(f a d 6)$. Aphid population growth

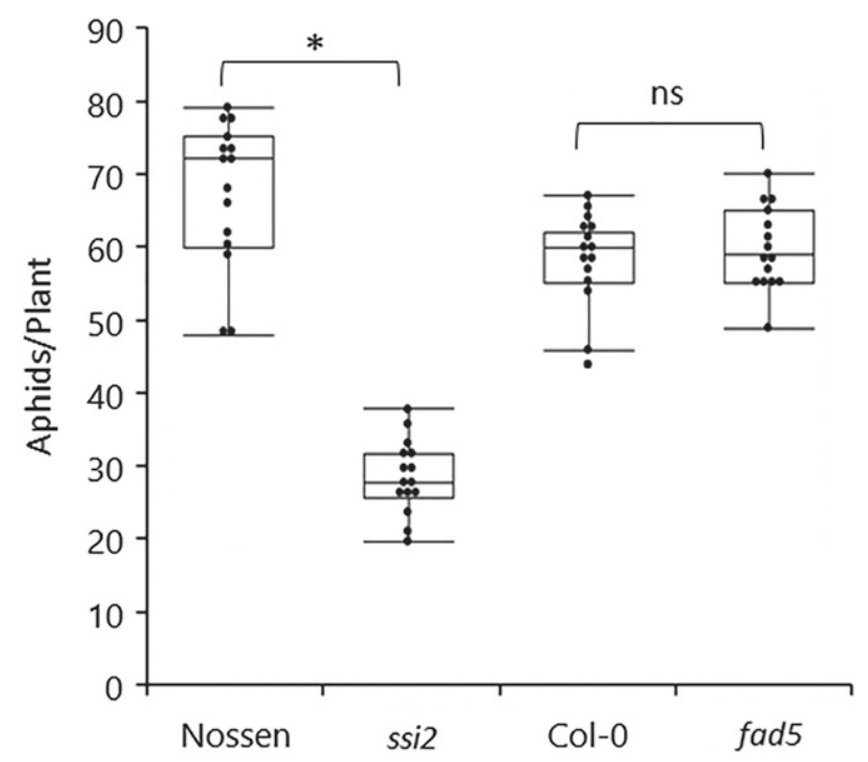

Fig. 2. Effects of desaturases that synthesize monoenoic FAs on aphid numbers. Plants were inoculated with three adults per plant, and the total number of live aphids was counted 7 days after inoculation on ssi2, fad5, and their respective wild-type controls (Nossen and Col-0, respectively) $(n=15)$. The data for each comparison was analyzed by Student's $t$ tests in JMP (SAS Institute). An asterisk (*) indicates statistically significant differences at $\alpha=0.05$; ns indicates no statistically significant differences at $\alpha=$ 0.05 . In the box plot, the bottom and top boundaries of the box indicate the 25th and 75th percentiles and a line within the box marks the median. Whiskers above and below the box indicate the 10th and 90th percentiles. 
was not significantly influenced by the $f a d 2$ mutation, which decreases C18:2 synthesis in the ER (Fig. 4). Compared with WT Col-0 controls, this mutant had approximately $51 \%$ lower C18:2 levels than WT plants, 10-fold higher C18:1 levels, and 19\% lower C18:3 levels (Fig. 5). C16 levels were unaffected by fad2, consistent with the fact that C16 FAs are not desaturated in the ER. The fad6 mutation also did not appear to have a strong impact on aphid numbers, because in assays in which the fad6 mutant showed normal growth and development, aphid numbers were comparable on fad6 and Col-0 (Fig. 4; Supplementary Fig. S7). Interestingly though, the fad6 mutant had much more variable growth than any other genotype in this study. In certain assays, fad6 formed smaller rosettes than the WT control Col-0 (Supplementary Fig. S4), and, in these assays, aphid numbers were lower on the mutant than on Col-0 (Supplementary Fig. S7). No significant differences in FA profiles were observed between small fad6 plants and fad6 plants that were comparable in size to Col-0 (Supplementary Fig. S5). This suggests that the reduced aphid numbers on small fad6 plants might be due to reduced plant health or surface area for colonization rather
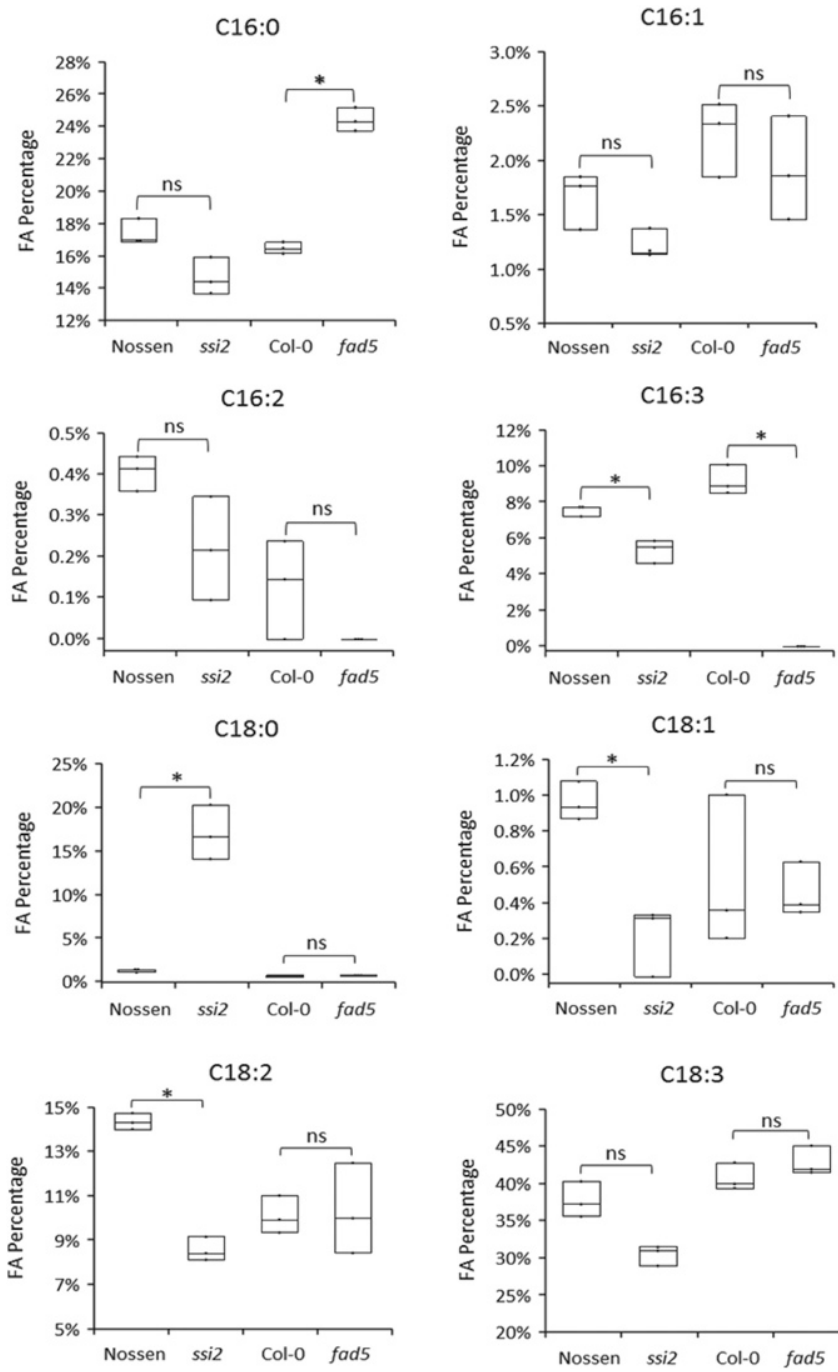

Fig. 3. Effects of ssi2 and fad5 on fatty acid (FA) profiles. Total FAs were extracted from foliage and were methylated, and fatty acid methyl esters were analyzed by gas chromatography-mass spectrometry. The data for each comparison was analyzed by Student's $t$ test in JMP (SAS Institute) $(n=3)$. Asterisks $(*)$ indicate statistically significant differences at $\alpha=$ 0.05 ; ns indicates no statistically significant differences at $\alpha=0.05$. In the box plot, the bottom and top boundaries of the box indicate the 25th and 75th percentiles and a line within the box marks the median. than to differences in FA profiles. Regardless of size, fad6 plants accumulated significantly more C18:1 and significantly less C18:3 and C16:3 than WT controls (Supplementary Fig. S5), which is consistent with the ability of the chloroplast-localized FAD6 enzyme to act on both $\mathrm{C} 16$ and $\mathrm{C} 18$ substrates (Browse et al. 1989). C18:2 levels in fad6 were comparable to levels in WT plants and significantly higher than levels in fad2 plants, indicating that FAD2 has a stronger impact on C18:2 accumulation than FAD6; however, neither mutation completely blocked dienoic FA accumulation (Fig. 5). These results indicate that partial loss of $\Delta-12$ FAD activity in the ER or chloroplast does not dramatically impact aphid population growth.

\section{Influence of $F A D 7$ and Glabral on aphid resistance.}

To confirm the effects of an $\omega-3$ FAD involved in trienoic FA synthesis on aphids, we also assessed whether aphids are impacted by the fad7-1 mutation, which impairs FAD7 function in the chloroplast. This mutation was generated in a Col-0 accession that also carried an additional mutation ( $g l l)$ at the GLABRAl locus, and Xia et al. (2010) reported that defects in systemic acquired resistance (SAR) that had previously been attributed to $\mathrm{fad} 7$ were, in fact, due to impairment of cuticle development by gll. To discriminate between the potential effects of $g l l$ versus $\mathrm{fad} 7 \mathrm{l}$ on aphids, aphid numbers on the original fad7-1 mutant line (referred to here as $f a d 7 g l l$ ) were compared with numbers on $g l l$ as well as to the WT genetic background Col-0. In addition, by crossing fad $7 \mathrm{gll}$ with Col-0, selfing the F1 generation, and screening subsequent

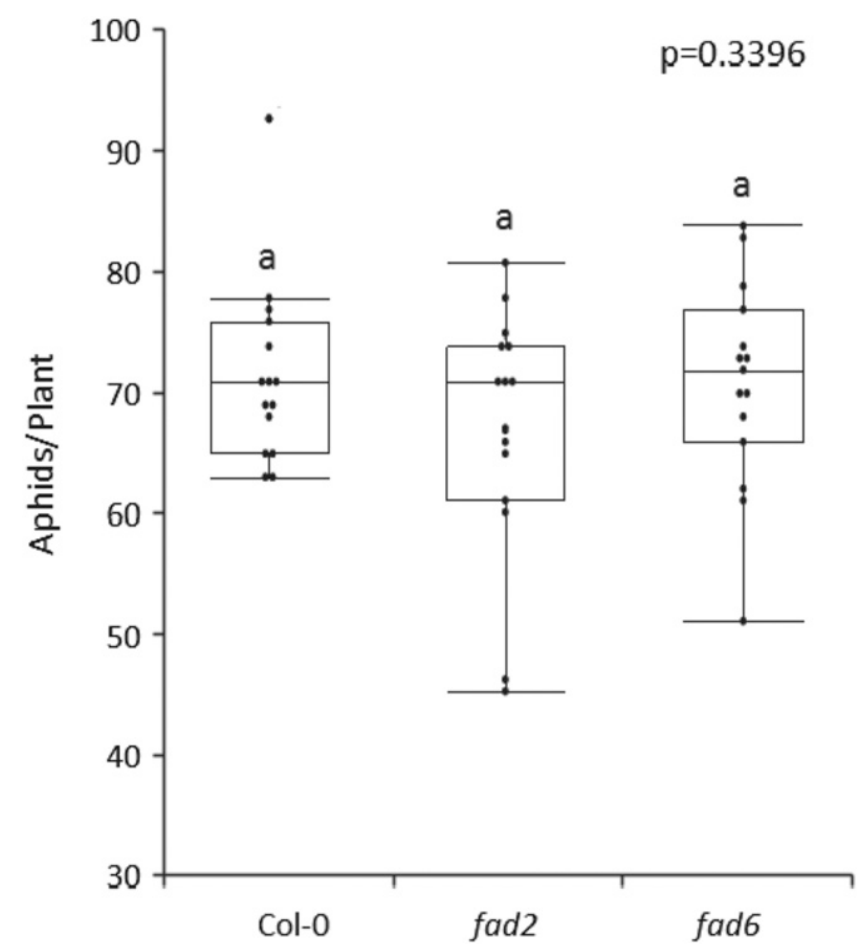

Fig. 4. Influence of $\Delta-12$ fatty acid desaturases (FADs) in the endoplasmic reticulum $(f a d 2)$ or chloroplast $(f a d 6)$ on aphid numbers. Plants were inoculated with three adults per plant, and the total number of live aphids was counted 7 days after inoculation on $\mathrm{fad} 2$, fad6, and their wild-type control (Col-0) $(n=15)$. The $P$ value reported is from a oneway analysis of variance (ANOVA) to determine if any differences exist among genotypes. Boxes having the same letter are not significantly different at $\alpha=0.05$, according to one-way ANOVA. In the box plot, the bottom and top boundaries of the box indicate the 25th and 75th percentiles and a line within the box marks the median. Whiskers above and below the box indicate the 10th and 90th percentiles. 
generations for both mutations, we also generated a line (referred to here as $f a d 7$ ) that lacks the $g l l$ mutation and is homozygous for the fad7-1 mutation (Vaughn et al. 2014). Aphid numbers on

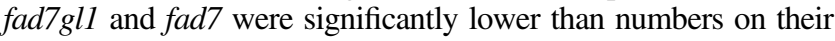
respective controls $g l l$ and Col-0 (Fig. 6A), confirming prior findings that loss of function of $F A D 7$ confers aphid resistance (Avila et al. 2012). Furthermore, there were no significant differences in population growth between $f a d 7 g l l$ and $f a d 7$ or between $g l l$ and Col-0 (Fig. 6A). The comparable aphid numbers observed on gll and Col-0 are also consistent with recent findings that the gll mutation does not strongly influence the incidence of naturally occurring aphid infestations on field-grown Arabidopsis (Sato et al. 2019). Thus, although GLABRA1-dependent cuticle formation may regulate SAR against bacteria (Xia et al. 2010), it does not impact local defenses against aphids in WT Arabidopsis or fad7 mutants. These data show that the fad7gll and fad7 mutants
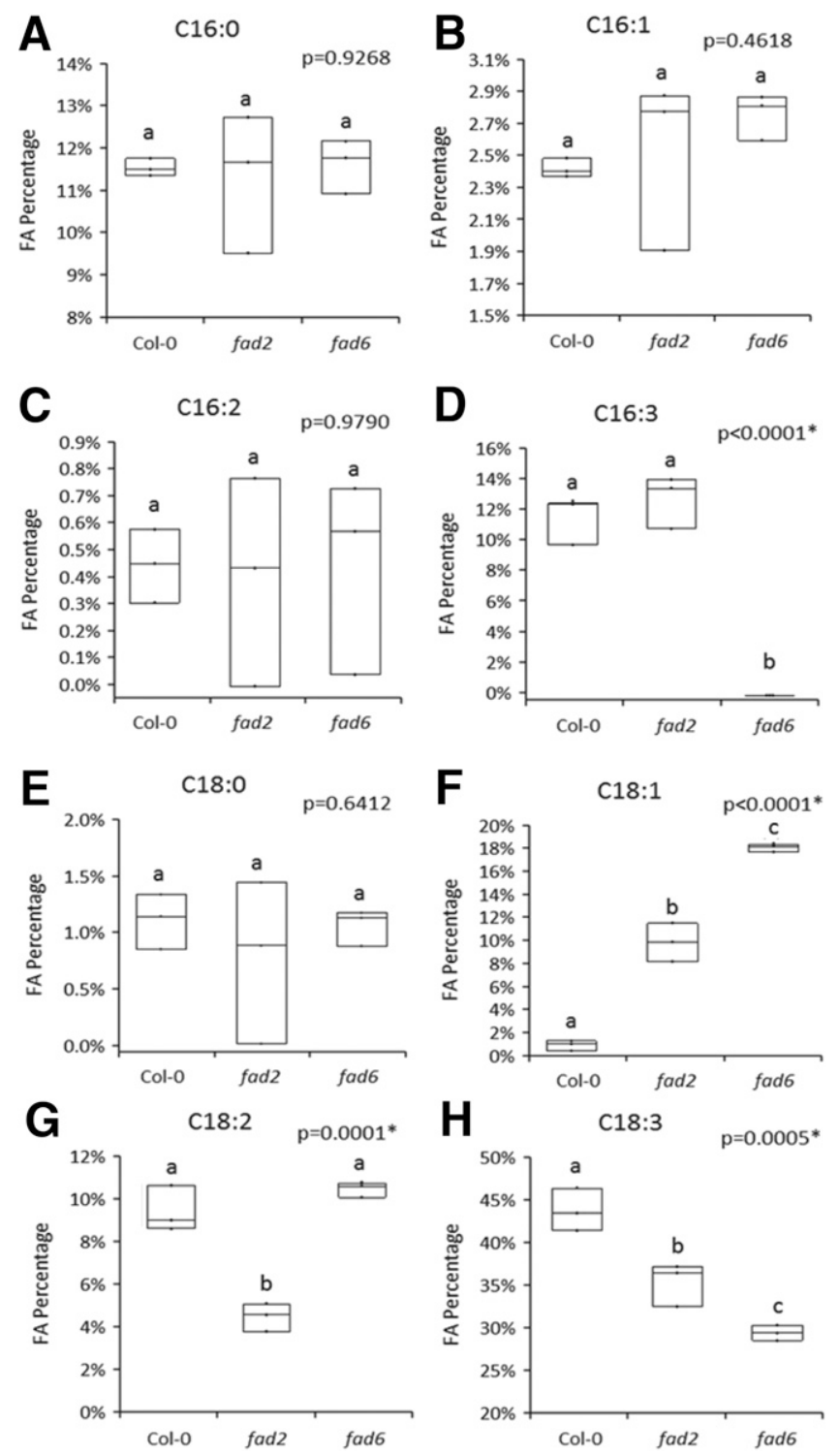

Fig. 5. Influence of $f a d 2$ and $f a d 6$ on fatty acid (FA) profiles. Total FAs were extracted from foliage and were methylated, and fatty acid methyl esters were analyzed by gas chromatography-mass spectrometry. The $P$ values reported are from one-way analyses of variance (ANOVA) to determine if any differences exist among treatments. Bars having the same letter are not significantly different at $\alpha=0.05$, according to $\mathbf{A}$, $\mathbf{B}, \mathbf{C}$, and $\mathbf{E}$, one-way ANOVA or $\mathbf{D}, \mathbf{F}, \mathbf{G}$, and $\mathbf{H}$, the Tukey-Kramer honest significant difference test $(n=3)$. In the box plot, the bottom and top boundaries of the box indicate the 25th and 75th percentiles and a line within the box marks the median. are equally resistant to green peach aphids and that the $g l l$ mutation did not significantly influence aphid numbers or contribute to aphid resistance in $\mathrm{fad} 7 \mathrm{gll}$. Loss of function of FAD7 in Arabidopsis appeared to affect population growth primarily by affecting adult fecundity rather than aphid survival; dead adults or juveniles were absent in most cages.

\section{Influence of other $\omega$-3 FADs in the chloroplast and ER on aphid resistance.}

Because loss of function of FAD7 results in aphid resistance, assays were conducted to determine whether other $\omega-3$ FADs that synthesize trienoic FAs in the chloroplast (FAD8) or the ER (FAD3) impacted aphids and to quantify their impacts on FA profiles. Aphid numbers on the fad 3 mutant were significantly lower than numbers on Col-0 and were comparable to numbers on both genotypes that carried the fad7 mutation (Fig. 6B). Similar to $\mathrm{fad} 7, \mathrm{fad} 3$ appeared to reduce offspring production but not increase mortality; only $15 \%$ of replicates had any mortality, and even these plants had only one or two dead aphids. These results demonstrate that loss of function of the ER-localized FAD3 decreases aphid fecundity and confers a comparable level of aphid resistance as loss of function of the chloroplast-localized FAD7. Whereas fad7 influenced the accumulation of both C16 and C18 FAs, fad3 affected only the C18 profile (Fig. 7). In fact, the only statistically significant difference in FA profiles between $\mathrm{fad} 3$ and the WT control Col-0 was an increase in C18:2 in fad3 (Fig. 7G). This data supports the hypothesis that
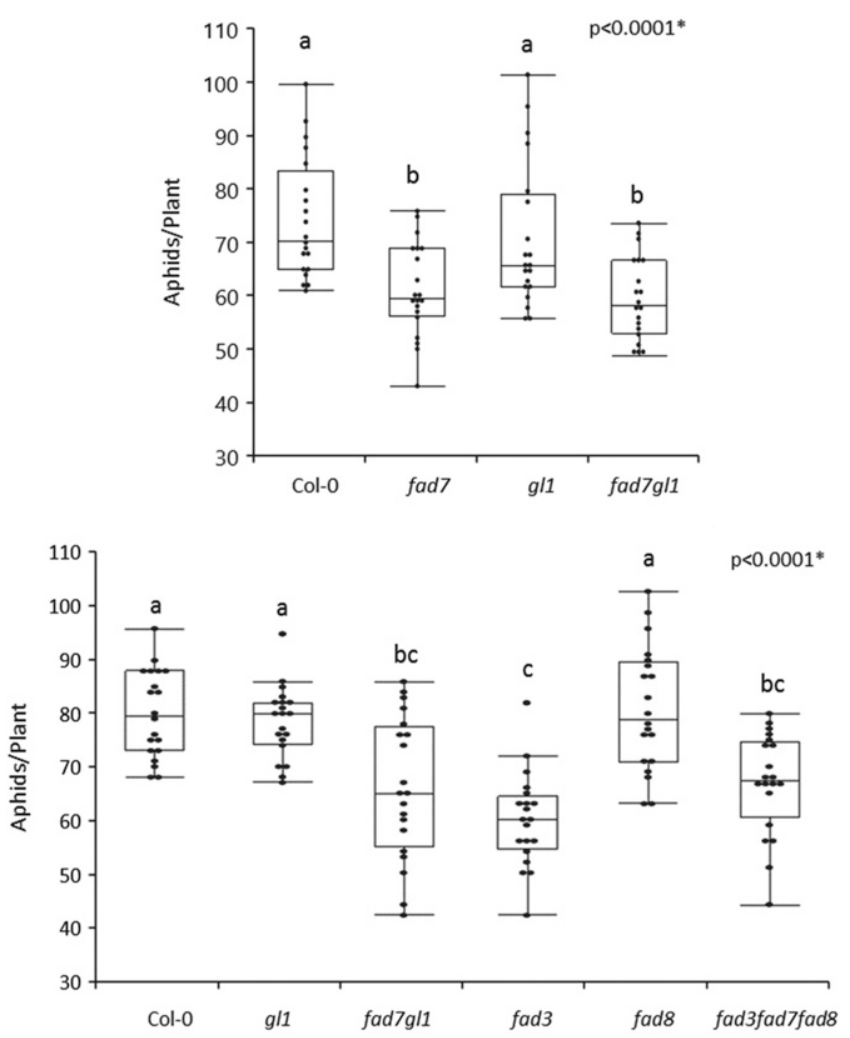

Fig. 6. Influence of the $g l l$ mutation and $\omega-3$ fatty acid desaturases (FADs) in the endoplasmic reticulum ( $\mathrm{fad} 3$ ) or chloroplast $(\mathrm{fad} 7 \mathrm{fad} 8)$ and on aphid numbers. Plants were inoculated with three adults per plant, and the total number of live aphids was counted 7 days after inoculation. The $P$ values reported are from one-way analyses of variance to determine if any differences exist among treatments. Boxes having the same letter are not significantly different at $\alpha=0.05$, according to the Tukey-Kramer honest significant difference test $(n=20)$. In the box plot, the bottom and top boundaries of the box indicate the 25th and 75th percentiles and a line within the box marks the median. Whiskers above and below the box indicate the 10th and 90th percentiles. 

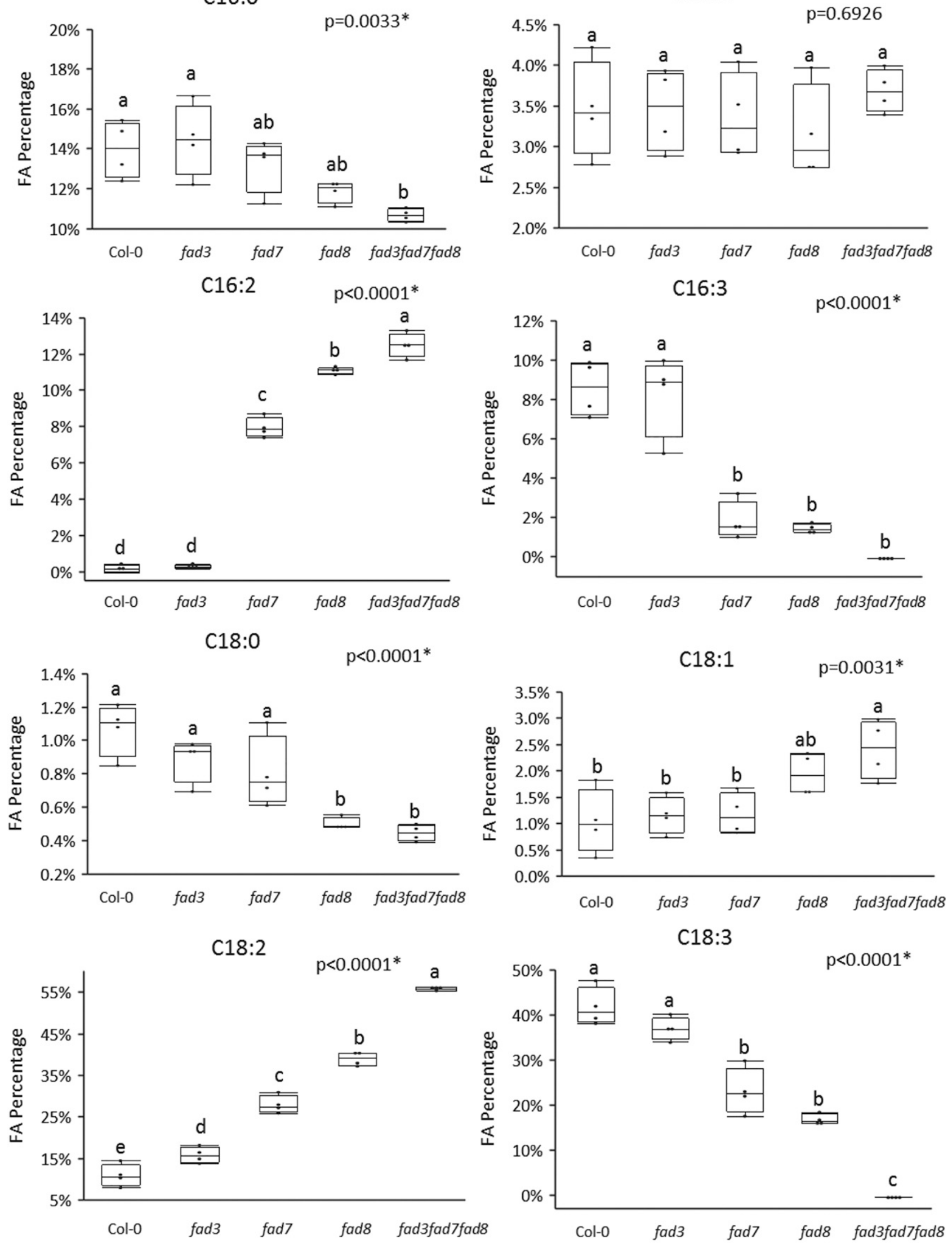

Fig. 7. Influence of $\omega-3$ fatty acid desaturases on fatty acid (FA) profiles. Total FAs were extracted from foliage and were methylated, and fatty acid methyl esters were analyzed by gas chromatography-mass spectrometry. The $P$ values reported are from one-way analyses of variance (ANOVA) to determine if any differences exist among treatments. Bars having the same letter are not significantly different at $\alpha=0.05$, according to one-way ANOVA (C16:1 panel) and the Tukey-Kramer honest significant difference test (all other panels) $(n=4)$. In the box plot, the bottom and top boundaries of the box indicate the 25 th and 75th percentiles and a line within the box marks the median. Whiskers above and below the box indicate the 10th and 90th percentiles. 
aphid population growth is influenced by $\mathrm{C} 18$ rather than $\mathrm{C} 16$ FAs. It also suggests that C18:2 may play a role in aphid resistance in $\mathrm{fad} 3$ and that resistance does not require significant depletion of trienoic FAs.

Compared with WT plants (Col-0), the fad8 mutation did not significantly impact aphids, whereas aphid numbers on both genotypes carrying the $\mathrm{fad} 7$ mutation were significantly lower than numbers on Col-0 (Fig. 6B). Although the $\mathrm{fad} 7 \mathrm{\text {and }} \mathrm{fad} 8$ mutants differed in their effects on aphids, their FA profiles were similar; the primary differences between these two mutants was that fad 8 had significantly lower levels of C18:0 than $\mathrm{fad} 7$ as well as significantly higher levels of $\mathrm{C} 16: 2$ and $\mathrm{C} 18: 2$ (Fig. 7C, G, and E). C18:1 levels were also more than 60\% higher in $\mathrm{fad} 8$ than in $\mathrm{fad}$, but whether this difference was statistically significant varied depending upon whether the data were analyzed with a Student's $t$ test $(P=0.0378)$ or the more conservative Tukey's $(P=$ 0.2055). The differences in aphid numbers between $\mathrm{fad} 7$ and $\mathrm{fad} 8$ suggest that aphid resistance in $f a d 7$ may be influenced by the abundance of more than one FA rather than by a single FA species.

Aphid populations on the fad $3 \mathrm{fad} 7 \mathrm{fad} 8$ triple mutant were significantly smaller than populations on Col-0 and were comparable to levels on the $f a d 3$ and $f a d 7 g l l$ lines, indicating that the resistance associated with the $\mathrm{fad} 3$ and $\mathrm{fad} 7$ mutations do not have additive effects on aphid populations (Fig. 6B). However, the $\mathrm{fad} 3 \mathrm{fad} 7 \mathrm{fad} 8$ triple mutant had significantly higher levels of C16:2 and C18:2 and significantly lower C18:3 than all other genotypes, demonstrating that the individual mutations had an additive effect on FA profiles (Fig. 7). Compared with $\mathrm{fad}$, the triple mutant also had significantly lower $\mathrm{C} 18: 0$ and significantly higher C18:1 (Fig. 7). Together, these results indicated that aphids are influenced by $\omega-3$ FADs in both the chloroplast and ER and may be affected by more than one C18 FA.

\section{Aphid numbers and FA profiles}

in double and triple mutants with impairments

in $\omega-3$ FAD and $\Delta-12$ FAD activity.

To assess whether dienoic FA accumulation is required for aphid resistance in the $f a d 7$ mutant, a fad $2 f a d 7$ mutant was developed through genetic crosses and CRISPR-Cas9 was employed to mutagenize FAD6 in a fad2fad7 genetic background, generating a fad2fad6fad7 mutant. The triple mutant line selected for this study had lost Cas 9 by segregation and carried a 38bp deletion in FAD6 in the same region as the fad6-1 mutation (Supplementary Fig. S2), at the putative active site of the FAD6 enzyme (Browse et al. 1989). Aphid numbers on fad2fad7 and fad2fad6fad7 were comparable to numbers on fad7 and were significantly lower than numbers on Col-0 (Fig. 8), indicating that $\mathrm{fad} 7$-mediated aphid resistance was intact in the double and triple mutants. C16:2 and C16:3 were undetectable in fad2fad6fad7, demonstrating that the mutation introduced into FAD6 blocked the function of the FAD6 enzyme (Supplementary Fig. S6). C18:1 levels in the triple mutant were comparable to levels observed in the fad6 mutant and significantly higher than all other genotypes. The ratio of C18:2 to C18:1 in fad2fad6fad7 was also significantly lower than in $\mathrm{fad} 7$ or WT plants and was comparable to levels observed in fad 2 and fad6 single mutants, while the ratio of C18:2 to C18:1 in the fad2fad7 mutant was intermediate between $\mathrm{fad} 7$ and $\mathrm{fad} 2$ (Supplementary Fig. S6). These observations reveal that the fad2 mutant and CRISPR Cas9 mutagenesis of FAD6 had additive inhibitory effects on the conversion of $\mathrm{C} 18: 1$ to $\mathrm{C} 18: 2$. Despite differences in the ratio of C18:2 to C18:1, however, total C18:2 abundance in the triple mutant remained elevated relative to the WT control Col-0 (Supplementary Fig. S6). These data suggest that the fad 2 mutation does not fully block FAD2 function and indicate that the impairments in $\Delta-12$ desaturase activity in fad2fad6fad7 were not sufficient to counteract the strong effects of $\mathrm{fad} 7$ on $\mathrm{C} 18: 2$ accumulation. Given that fad $2 \mathrm{fad} 6 \mathrm{fad} 7 \mathrm{\text {was }}$ resistant to aphids but lacked detectable levels of $\mathrm{C} 16: 2$, these results also indicate that the heightened $\mathrm{C} 16: 2$ accumulation observed in $\mathrm{fad} 7$ is not required for aphid resistance.

\section{DISCUSSION}

Population growth of a generalist herbivore, the green peach aphid, are reduced on Arabidopsis lines with impairments in $F A D 7, F A D 3$, and SSI2. This indicates that certain FADs promote

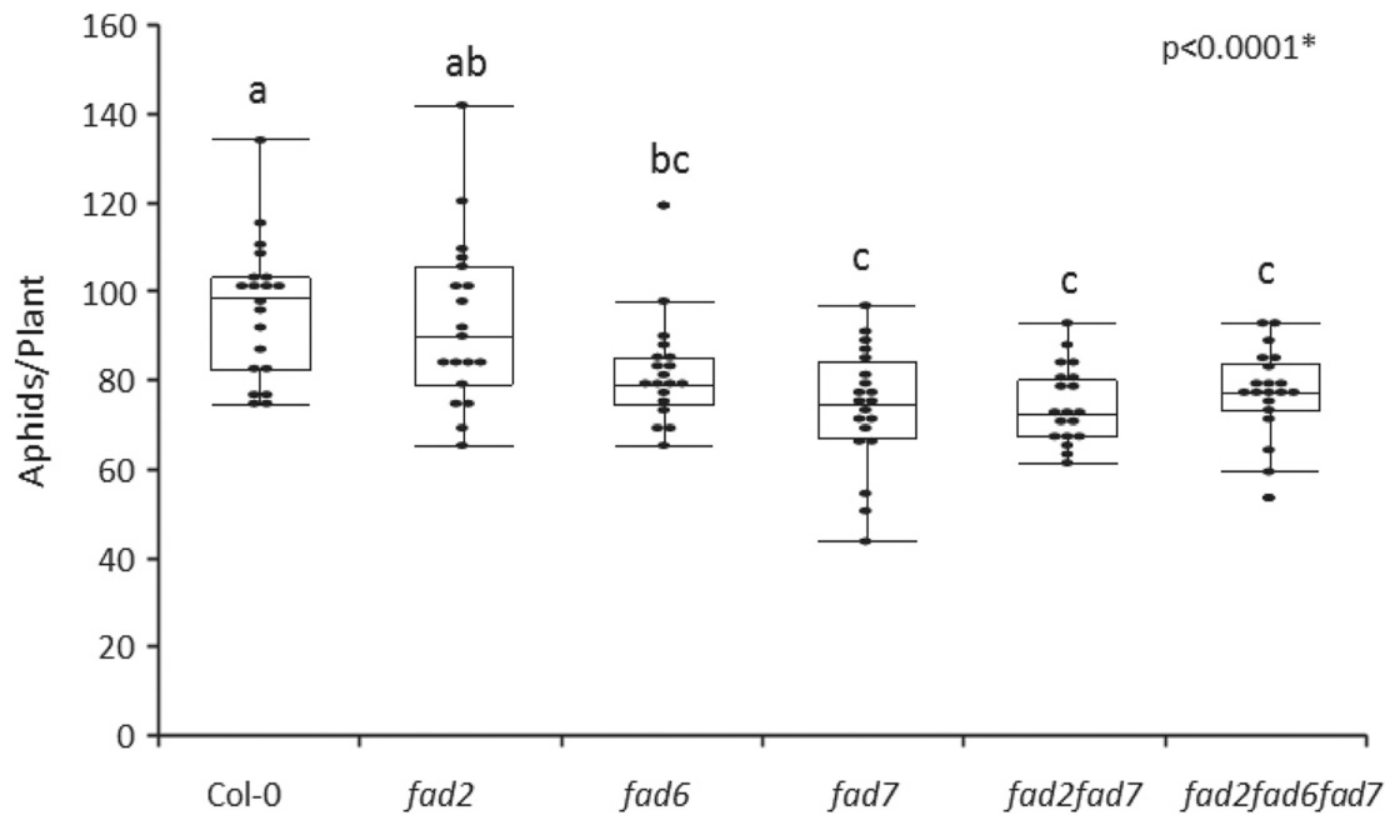

Fig. 8. Combined influence of an $\omega-3$ fatty acid desaturase (FAD) (fad7) and $\Delta-12$ FADs (fad2 and fad6) on aphid numbers. Aphid population growth on Arabidopsis lines impaired in conversion of dienoic to trienoic fatty acids. The $P$ value reported is from a one-way analysis of variance to determine if any differences exist among treatments. Boxes having the same letter are not significantly different at $\alpha=0.05$, according to the Tukey-Kramer honest significant difference test $(n=20)$. In the box plot, the bottom and top boundaries of the box indicate the 25 th and 75 th percentiles and a line within the box marks the median. Whiskers above and below the box indicate the 10th and 90th percentiles.

Vol. 34, No. 6, $2021 / 697$ 
susceptibility to aphids. Several lines of evidence suggest that the effects of these desaturases on aphids are mediated through desaturation of $\mathrm{C} 18$ rather than C16 FAs. Although the fad7 mutant accumulates higher-than-normal levels of C16:2 as well as $\mathrm{C} 18: 2$, a triple mutant line that lacks detectable levels of C16:2 ( $f a d 2 f a d 6 f a d 7)$ nonetheless retains levels of aphid resistance comparable to those of fad7. Aphid numbers are also unaffected by the fad5 mutation that inhibits $\mathrm{C} 16: 1$ synthesis; in contrast, aphid population growth is diminished by the ssi2 mutation, which inhibits C18:1 synthesis. Furthermore, the fad 3 mutant had levels of resistance comparable to those of the fad7 mutant even though fad3 only impacts C18 levels. Thus, aphid resistance is more strongly associated with differences in $\mathrm{C} 18$ abundance than $\mathrm{C} 16$ abundance.

A comparison of the multiple desaturase mutants examined in this study suggests that aphids are impacted by more than one C18 FA (a summary of all mutants is provided in Supplementary Fig. S8). A previous study on ssi2 hypothesized that aphid resistance in this mutant is due to decreased levels of C18:1 (Louis et al. 2010a). Except for ssi2, all other genotypes that displayed aphid resistance in the current study had higher than normal levels of C18:2; in fact, this was the only FA that differed significantly between $f a d 3$ and WT controls. Therefore, C18:2 may potentially contribute to aphid resistance in certain genotypes. However, elevated C18:2 levels alone were not sufficient to confer resistance to fad8. Notably, this mutant had nearly twice as much $\mathrm{C} 18: 1$ and half as much C18:0 than WT plants; possibly the effects of heightened C18:2 accumulation in this genotype were countered by elevated C18:1 levels or other changes in the FA profile. Together, these data suggest that aphids are impacted by more than one $\mathrm{C} 18 \mathrm{FA}$ and that C18:2 may promote aphid resistance whereas $\mathrm{C} 18: 1$ promotes susceptibility.

The observation that the fad 3 mutation reduces aphid numbers also demonstrates that aphids are impacted by desaturases in the ER as well as by the chloroplastic desaturases disrupted by the ssi 2 and fad 7 mutations. This may be due to the extensive metabolic exchange between the ER and chloroplast. The ER forms numerous points of physical contact with the outer membranes of plastids, and these membrane contact sites are thought to facilitate nonvesicular lipid transfer (Michaud and Jouhet 2019; Wang and Benning 2012). Hemifusion between the membranes of the ER and chloroplast could, theoretically, also enable enzymes from one organelle to act on substrates in another organelle (Mehrshahi et al. 2014). While the mechanisms of interorganellar exchange are not fully understood, it is well-established that the pathways for lipid biosynthesis in the chloroplast and the ER are intertwined (Fig. 1) (Karki et al. 2019; Mehrshahi et al. 2014; Somerville and Browse 1996). Chloroplast membranes are composed primarily of galactolipids, and the diacylglycerol required for their synthesis in the chloroplast is derived entirely (in $\mathrm{C} 18$ plants) or in part (in $\mathrm{C} 16$ plants) from phosphatidylcholine (PC) or PC-derived intermediates imported from the ER (Michaud and Jouhet 2019). This PC pool may be desaturated by FAD3 prior to export (Karki et al. 2019); thus, FAD3 activity in the ER may impact the FA pool in the chloroplast. Phospholipid synthesis in the ER also requires FAs synthesized in chloroplasts, including C18:1, desaturated by the FAB2/SSI2 gene product (Michaud and Jouhet 2019). Moreover, chloroplast-localized $\omega-3$ desaturases have been reported to contribute to C18:3 accumulation in lipids synthesized in the ER in olive and birch (Hernández et al. 2016; Martz et al. 2006) and may also partially compensate for null mutations in FAD3 in Arabidopsis (Browse et al. 1993). Thus, the influence of FAB2/ SSI2, FAD7, and FAD3 on plant-aphid interactions could be mediated through their impacts on lipid metabolism in the chloroplast, the ER, or both cellular compartments. Furthermore, an emerging body of evidence suggests that lipid signaling between the ER and chloroplast is important to plant stress responses (Liu and Li 2019; Michaud and Jouhet 2019). ER-chloroplast membrane contact sites have been identified as a location for several lipid transfer proteins involved in SAR to pathogens, including AZELAIC ACID INDUCED 1 (AZI1), EARLY ARABIDOPSIS ALUMINUM INDUCED 1 (EARLI1), and DEFECTIVE IN INDUCED RESISTANCE 1 (DIR1) (Cecchini et al. 2015). Moreover, the ssi2 mutation, which constitutively activates multiple components of SAR, also constitutively activates the expression of a marker (BIP3) of the unfolded protein response, a stress-responsive intracellular signaling pathway initiated in the ER (Iwata et al. 2018). Potentially, altered signaling between the chloroplast and ER may contribute to aphid resistance in one or more of the ssi2, fad3, or $\mathrm{fad} 7$ mutants.

The effects of FADs on aphids indeed appear to be due to their influence on defensive signaling rather than to direct effects of FAs on aphids. It is unlikely that decreased desaturase activity would decrease the nutritional quality of plants for aphids because phloem sap is reported to contain primarily unsaturated FAs (Madey et al. 2002). Instead, the fad7 mutation likely promotes defense signaling and possibly also secondary metabolite production against aphids. Similar to fad7 in Arabidopsis, the spr2 mutation in SIFAD7 in tomato confers strong resistance to the potato aphid Macrosiphum euphorbiae and this resistance requires salicylic acid (SA) signaling; resistance is diminished by the NahG transgene, which suppresses SA accumulation, and by transient silencing of NONEXPRESSOR OF PATHOGENESIS-RELATED GENES 1 (NPRl), a key regulator of many SA-dependent defenses (Avila et al. 2012). Aphid resistance in spr2 is also diminished by suppressing expression of LIPOXYGENASE1 (LOX1), which can act on C18:2 or other FAs to generate multiple oxylipins (Avila et al. 2013). Potentially the altered FA content of FAD7-impaired plants may result in a shift in the oxylipin profile generated by LOX1, favoring compounds with activity against aphids. Similarly, resistance to rice blast in $O S F A D 7-$ and $O s$ $F A D 8$-silenced rice lines is associated with enhanced hydroperoxides and hydroxides derived from C18:2, indicating that C18:2derived oxylipins may be important to resistance (Yara et al. 2007, 2008). Further work is needed to characterize the mechanisms of aphid resistance in plants with impaired FAD7 activity, and to determine whether FAD3 influences plant-aphid interactions through similar or divergent pathways.

Similar to loss of function of FAD7, the ssi2 mutation also impacts aphids by modifying plant immunity. Although petiole exudates from ssi 2 inhibit aphid growth on artificial diets, this inhibitory effect is not due to direct effects of FAs because the addition of C18:0 or C18:1 to an artificial diet has no effect on aphid population growth (Louis et al. 2010a). Instead, aphid resistance in ssi2 depends upon altered defense signaling, although it has different signaling requirements than $\mathrm{fad} 7$-mediated aphid resistance (Louis et al. 2010a and b; Pegadaraju et al. 2005). In contrast to aphid resistance in FAD7-impaired tomato, aphid resistance in ssi2 is not impaired by the $N a h G$ transgene or a null mutation in NPRl (Pegadaraju et al. 2005). Aphid resistance in ssi2 does, however, require an aphid-inducible lipase (MPL1) and PHYTOALEXIN DEFICIENT4, suggesting that this resistance is due to altered lipid signaling (Louis et al. 2010a and b). Furthermore, analysis of other ssi2-dependent phenotypes indicate that this mutation promotes defense signaling by releasing nitric oxide (NO) production from negative regulation by $\mathrm{C} 18: 1$. The ssi2 mutation causes increased constitutive SA accumulation and enhanced resistance to multiple pathogens, including the oomycete Peronospora parasitica and the bacterial pathogen Pseudomonas syringae (Kachroo et al. 2001; Shah et al. 2001). Second-site mutations that restore C18:1 accumulation in ssi2 inhibit constitutive SA accumulation and restore WT levels of susceptibility to Peronospor parasitica (Kachroo et al. 2003, 
2004). C18:1 acts as a negative regulator of NO signaling by binding to NITRIC OXIDE ASSOCIATED1 (NOA1) and promoting its degradation; conversely, C18:1 depletion in ssi2 promotes NO production and consequent defense signaling (Mandal et al. 2012). Furthermore, a null mutation in NOAl compromises the enhanced resistance of the ssi2 mutant to Pseudomonas syringae (Mandal et al. 2012). Based on these observations of pathogen resistance in ssi2, aphid resistance in this mutant may also depend upon altered defensive signaling mediated by C18:1 depletion. Notably, NO has been implicated in Russian wheat aphid resistance responses in wheat, and exogenous NO reduces aphid feeding on pea (Moloi and van der Westhuizen 2014; Woźniak et al. 2017). Therefore, further work is warranted to determine if NO signaling contributes the effects of desaturases on aphid numbers.

In summary, plant desaturases in both the chloroplast and ER promote susceptibility to aphids. FAD7 appears to predispose plants to aphid attack by inhibiting SA-dependent defenses, while the stearoyl-acyl carrier protein desaturase encoded by FAB2/ SSI2 may facilitate aphid population growth by suppressing NO signaling; in both cases, these effects are likely mediated through the influence of desaturases on C18 FA profiles and lipid signaling. The potential role of desaturases in stress-responsive signaling between the ER and chloroplast also merits further study. These results shed light on the influence of plant primary metabolism on plant immunity against aphids, an important group of insect pests whose mode of parasitism bears many similarities to plant pathogens (Kaloshian and Walling 2005).

\section{MATERIALS AND METHODS}

\section{Plant materials.}

Seeds for Arabidopsis thaliana lines carrying the fad2-1, fad3-2, fad5-1, and fad6-1 mutations as well as the WT Columbia ecotype (Col-0, accession CS70000) were obtained from the Arabidopsis Biological Resource Center. Seeds for gll (GLABRA1), Nossen, and ssi2 (SUPPRESSOR OF SALICYLIC ACID INSENSITIVITY2) were donated by J. Shah, University of North Texas, Denton, TX, U.S.A., who also provided seeds for the fad7-1 mutant, which also carries the gll mutation (Xia et al. 2010); for clarity, fad7-1 is referred to here as fad7gll. The triple mutant line fad $3 f a d 7 f a d 8$ was obtained from J. Browse, Washington State University, Pullman, WA, U.S.A. A mutant line (referred to here as $f a d 7$ ) that carries only the fad7-1 mutation without the gll mutation was developed by Vaughn et al. (2014). The fad2fad7 mutant was developed by crossing fad2-1 and fad7-1gll (described below). Seeds were surface-sterilized and plated on Murashige and Skoog germination medium (PhytoTechnology Laboratories, Shawnee Mission, KS, U.S.A.), then, were vernalized for 3 days at $4^{\circ} \mathrm{C}$. After transfer to soil (a 4:3:2 mixture of peat, vermiculite, and perlite) supplemented with 15-9-12 Osmocote Plus fertilizer (Scotts-MiracleGro Company, Marysville, OH, U.S.A.), plants were maintained in a Conviron growth chamber (Controlled Environments Inc., Pembina, ND, U.S.A.) at $23^{\circ} \mathrm{C}$, with a L13: D11 photoperiod and around $65 \%$ relative humidity. The plants were fertilized weekly with Miracle Gro all-purpose plant food (Scotts-MiracleGro Company) and were watered as needed with tap water.

\section{Development of an Arabidopsis fad $2 \mathrm{fad} 7$ double mutant line.}

The Arabidopsis double mutant $\mathrm{fad} 2 \mathrm{fad} 7 \mathrm{was}$ developed by crossing fad $7 \mathrm{gll}$ (maternal parent) and fad 2 (pollen donor) and screening the F2 generation for plants homozygous for both mutations, using allele-specific PCR primers (Flattmann 2015). To select against the gll mutation, only progeny with trichomes were saved, because plants homozygous for gll lack trichomes (Oppenheimer et al. 1991). DNA was extracted using an extraction buffer that was made by diluting Edwards solution $(200 \mathrm{mM}$ Tris- $\mathrm{HCl}$ [pH 7.5], $250 \mathrm{mM} \mathrm{NaCl}, 25 \mathrm{mM}$ EDTA, and $0.5 \%$ sodium dodecyl sulfate) 10 -fold with TE buffer $(10 \mathrm{mM}$ Tris- $\mathrm{HCl}[\mathrm{pH}$ 8] and 1 mM EDTA) (Edwards et al. 1991; Kasajima et al. 2004). PCR primers were designed to distinguish between the WT AtFAD7 allele and the fad7-1 mutation reported by Xia et al. (2010) and between the WT AtFAD2 allele and the fad2-1 mutant sequence reported by Zhang et al. (2012) (Supplementary Fig. S1). The primers are

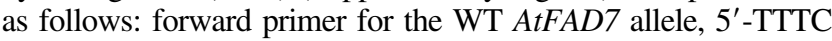
AGTGGGCTCGAAGTCC-3' and reverse primer, 5'-ATCTG CGGGAAAAGATGATG-3'; forward primer for the fad7-1 mutant allele, $5^{\prime}$-TTTCAGTGGGCTCGAAGACT-3' and reverse primer, 5' -ATCTGCGGGAAAAGATGATG-3'; forward primer for the WT Atfad2-1 allele, 5'-CCTAACTGGTATCTGGGTCA TAG- $3^{\prime}$ and reverse primer, 5'-AACGAGGGATGAGTGTGC TG-3'; forward primer for the fad2-1 mutant allele, 5'-CCTAAC TGGTATCTGGGTCACAA-3' and reverse primer, 5' -AACGA GGGATGAGTGTGCTG-3'. Touchdown PCR was performed to increase amplification sensitivity and specificity. For the WT AtFAD7 allele, initial denaturation $=95^{\circ} \mathrm{C}$ for $5 \mathrm{~min}$; phase $\mathrm{I}=$ $95^{\circ} \mathrm{C}$ for $45 \mathrm{~s}, 65$ to $58^{\circ} \mathrm{C}$ for $45 \mathrm{~s}$ (reducing $1^{\circ} \mathrm{C}$ per cycle), and $72^{\circ} \mathrm{C}$ for $45 \mathrm{~s}$; phase $\mathrm{II}=95^{\circ} \mathrm{C}$ for $45 \mathrm{~s}, 57^{\circ} \mathrm{C}$ for $45 \mathrm{~s}$, and $72^{\circ} \mathrm{C}$ for $45 \mathrm{~s}$ ( 20 cycles); and a final extension at $72^{\circ} \mathrm{C}$ for $5 \mathrm{~min}$. For the Atfad7-1 mutation, the same program was used but with annealing temperatures of 65 to $56^{\circ} \mathrm{C}$ in phase $\mathrm{I}$ and $55^{\circ} \mathrm{C}$ in phase II. For the WT AtFAD2 allele, the annealing temperatures were adjusted to 64 to $57^{\circ} \mathrm{C}$ in phase I and $56^{\circ} \mathrm{C}$ in phase II. For the fad2-1 mutant allele, annealing temperatures of 60 to $53^{\circ} \mathrm{C}$ in phase I and $52^{\circ} \mathrm{C}$ in phase II were used. PCR products were visualized by agarose gel electrophoresis.

\section{CRISPR-Cas9 mutagenesis of FAD6.}

A CRISPR construct encoding four single guide RNA (sgRNA) to knock out FAD6 in Arabidopsis was generated according to the protocols developed by Ma et al. (2015). Each sgRNA contained the Arabidopsis U6-29 promoter, a 20-bp target sequence from AtFAD6, a guide RNA sequence, and the Pol III terminator (Supplementary Fig. S2A). The four target sequences within AtFAD6 were selected using the CRISPRPLANT online web tool and were tested to minimize off target hybridization (CRISPR/Cas9 target online predictor) and optimize GC content (40 to 60\%) and secondary structure formation (Zuker 2003). Double-stranded sgRNA adaptors were generated by combining $1.0 \mu \mathrm{M}$ of forward primer with an AATG overhang on the $5^{\prime}$ end and $1.0 \mu \mathrm{M}$ reverse primer with an AAAC overhang in $0.5 \times \mathrm{TE}$ solution, incubating in a thermal cycler at $90^{\circ} \mathrm{C}$ for $10 \mathrm{~min}$, and returning to room temperature with a decrease of $0.2^{\circ} \mathrm{C}$ per second. To generate each of the sgRNA clones, double-stranded sgRNA adaptors (Supplementary Table S1) were ligated into the pYLsgRNA-AtU6-29 vector (Addgene plasmid 66203) at Bsa I restriction sites downstream of the Arabidopsis U6-29 promoter. Two PCR reactions were performed to amplify i) the U6-29 promoter and the sgRNA sequence, and ii) the sgRNA sequence and the Pol III terminator sequence. Overlap PCR was performed with primers containing BSA I and the four unique nucleotide sequences to assemble each of the four sgRNA cassettes. Finally, all four sgRNA cassettes were inserted together into the plant expression vector pYLCRISPR/Cas9Pubi-H by the Golden Gate method (Engler et al. 2008). The design of the construct containing all four sgRNAs is represented in Supplementary Figure S2B. The pYLCRISPR/Cas9Pubi-H vector (Addgene plasmid 66187) also carries Cas9 driven by a maize ubiquitin promoter and a hygromycin selection marker driven by the double cauliflower mosaic virus $35 \mathrm{~S}$ promoter. The resulting final vector $\mathrm{pYL}$ CRISPR/Cas9Pubi-H-AtFAD6 $\mathrm{T}_{1} \mathrm{~T}_{2} \mathrm{~T}_{3} \mathrm{~T}_{4}$ was transformed into Escherichia coli and the plasmid was checked for the presence 
of the insert by PCR and sequencing. The plasmid with the correct orientation of sgRNAs was transformed in Agrobacterium tumefaciens GV3101. This construct was introduced into the Arabidopsis fad2fad7 double mutant by Agrobacterium-mediated transformation according to the methods of Bechtold and Bouchez (1995). To screen for transformants, surface-sterilized T0 seeds were plated on 1× Murashige and Skoog plates containing a final concentration of $25 \mu \mathrm{g} / \mathrm{ml}$ of hygromycin and hygromycin-resistant seedlings were selected according to Harrison et al. (2006). DNA from hygromycin-resistant plants was extracted with Edwards solution (Kasajima et al. 2004), and PCR was performed to confirm the presence of the $\operatorname{Cas} 9$ gene. Lines positive for $\operatorname{Cas} 9$ were then screened to identify mutations using two primer sets, one to amplify the region flanking targets 1 and 2 and the other to amplify a region flanking targets 3 and 4 (Supplementary Table S1). Deletions were identified by gel electrophoresis, and amplicons were eluted from the gel and were sequenced to confirm mutations. Lines that showed heterozygous deletions were propagated and screened in subsequent generations to select for lines that were homozygous for a deletion in FAD6 and that had lost the $\operatorname{Cas} 9$ gene by segregation. The line utilized for this study (fad2fad6fad7) was confirmed to carry a 38-bp deletion between targets 3 and 4 (Supplementary Fig. 2C and D), which includes the site mutagenized in the fad6-1 line (Zhang et al. 2009).

\section{Aphid bioassays.}

The green peach aphid Myzus persicae was reared on cabbage (Brassica oleracea var. Joychoi) at about $24^{\circ} \mathrm{C}$ and with a 16$\mathrm{h}$ light photoperiod. To produce cohorts of age-synchronized aphids for bioassays, cabbage plants were inoculated with wingless adult aphids that were allowed to larviposit on plants for 24 h. Adult aphids were removed after $24 \mathrm{~h}$, and the age-synchronized nymphs were monitored daily to track development. Apterous adult aphids for bioassays were collected within $24 \mathrm{~h}$ of emergence to adulthood. Arabidopsis plants at developmental stage 5.1 (Boyes et al. 2001) were challenged with three apterous adults per plant, using sleeve cages that covered the entire plant (15 to 20 replicate plants per genotype). After infestation, plants were maintained in a Conviron growth chamber $\left(23^{\circ} \mathrm{C}\right.$, $65 \%$ relative humidity, $13-\mathrm{h}$ light and $11-\mathrm{h}$ dark photoperiod), and the numbers of live and dead adults and offspring on each plant were scored 7 days after infestation.

\section{FA profile analysis.}

To assess the FA profiles of the mutant genotypes used in aphid bioassays, uninfested plants from each genotype were grown in empty sleeve cages and leaf tissue for FA analysis was collected from these plants 8 days after the beginning of the bioassay, when the plants were at developmental stage 6.0 (Boyes et al. 2001). Uninfested tissues were selected for analysis because preliminary measurements of FAs in WT plants (Col-0) indicated that the levels of aphid infestation observed in our bioassays did not significantly impact FA abundance in foliage (Supplementary Fig. S3). Leaf tissue was weighed and flashfrozen in liquid nitrogen (three to four replicates per genotype). Total FAs were extracted and methylated using an acid-catalyzed transmethylation protocol (Li-Beisson et al. 2013) combined with a purification method by Shipley et al. (1993). Fatty acid methyl esters (FAMEs) were dried down under nitrogen flow and were dissolved into hexane, then, were transferred into inserts of gas chromatography (GC) autosampler vials (HP 6890; Agilent). One microliter of each sample was injected in the split mode with an autosampler (Agilent Technologies) for GC-mass spectrometry (MS) analysis. The GC-MS system used a FAMEWAX column (30 $\mathrm{m} \times 0.25 \mathrm{~mm}$ with a $0.25 \mu \mathrm{m}$ film thickness; Restek). The initial temperature of the $\mathrm{GC}$ oven was $130^{\circ} \mathrm{C}$, which was ramped to $225^{\circ} \mathrm{C}$ at a rate of $7^{\circ} \mathrm{C} / \mathrm{min}$, held for $12 \mathrm{~min}$. FAMEs were identified by comparing retention times and mass spectra to external standards (Nuchek mixture reference; Nu-Chek Prep, Inc.) and were quantified based on an internal standard (pentadecanoic acid, C15:0; Nu-Chek Prep, Inc.) that does not naturally occur in Arabidopsis. The abundance of each FA was expressed relative to the total moles of FA in each sample (i.e., in mol \%); this compensates for any sample-to-sample differences in FA extraction efficiency.

\section{Statistical analysis.}

All the statistical analysis was done with JMP (SAS Institute Inc.). The data were analyzed by one- or two-way analysis of variance, and, when appropriate, means were separated using the Tukey-Kramer honest significant difference (for comparisons of multiple treatment groups) or Student's $t$ test (for comparisons of paired genotypes).

\section{ACKNOWLEDGMENTS}

We thank J. Shah at the University of North Texas and J. Browse at the Washington State University for providing seeds. We also thank R. Liyanage for assistance with GC-MS and J. Kivett for support with growth chamber and greenhouse facilities.

\section{AUTHOR-RECOMMENDED INTERNET RESOURCES}

Addgene: https://www.addgene.org/66203 and https://www.addgene.org/66187

CRISPR-PLANT online web tool: https://www.genome.arizona.edu/crispr

CRISPR/Cas9 target online predictor: https://cctop.cos.uni-heidelberg.de

\section{LITERATURE CITED}

Arondel, V., Lemieux, B., Hwang, I., Gibson, S., Goodman, H. M., and Somerville, C. R. 1992. Map-based cloning of a gene controlling $\omega-3$ fatty acid desaturation in Arabidopsis. Science 258:1353-1355.

Avila, C. A., Arévalo-Soliz, L. M., Jia, L., Navarre, D. A., Chen, Z., Howe, G. A., Meng, Q. W., Smith, J. E., and Goggin, F. L. 2012. Loss of function of FATTY ACID DESATURASE7 in tomato enhances basal aphid resistance in a salicylate-dependent manner. Plant Physiol. 158:2028-2041.

Avila, C. A., Arévalo-Soliz, L. M., Lorence, A., and Goggin, F. L. 2013. Expression of $\alpha$-DIOXYGENASE 1 in tomato and Arabidopsis contributes to plant defenses against aphids. Mol. Plant-Microbe Interact. 26:977-986.

Baker, R. 1994. Insect and related pests of flowers and foliage plants: Some important, common, and potential pests in the southeastern United States. Publication AG-136, revised ed. North Carolina Cooperative Extension Service, Raleigh, NC, U.S.A.

Bass, C., Puinean, A. M., Zimmer, C. T., Denholm, I., Field, L. M., Foster, S. P., Gutbrod, O., Nauen, R., Slater, R., and Williamson, M. S. 2014. The evolution of insecticide resistance in the peach potato aphid, Myzus persicae. Insect Biochem. Mol. Biol. 51:41-51.

Bechtold, N., and Bouchez, D. 1995. In planta Agrobacterium-mediated transformation of adult Arabidopsis thaliana plants by vacuum infiltration. Pages 19-23 in: Gene Transfer to Plants. I. Potrykus and G. Spangenberg, eds. Springer, Berlin.

Blackman, R. L., and Eastop, V. F. 1994. Aphids on the World's Trees: An Identification and Information Guide CAB International, Wallingford, U.K.

Blackman, R. L., and Eastop, V. F. 2000. Aphids on the world's crops, 2nd ed. John Wiley \& Sons, Chichester, U.K.

Blée, E. 2002. Impact of phyto-oxylipins in plant defense. Trends Plant Sci. 7:315-322

Boyes, D. C., Zayed, A. M., Ascenzi, R., McCaskill, A. J., Hoffman, N. E., Davis, K. R., and Görlach, J. 2001. Growth stage-based phenotypic analysis of Arabidopsis: A model for high throughput functional genomics in plants. Plant Cell 13:1499-1510.

Browse, J., Kunst, L., Anderson, S., Hugly, S., and Somerville, C. 1989 A mutant of Arabidopsis deficient in the chloroplast 16:1/18:1 desaturase. Plant Physiol. 90:522-529. 
Browse, J., McConn, M., James, D., Jr., and Miquel, M. 1993. Mutants of Arabidopsis deficient in the synthesis of $\alpha$-linolenate. Biochemical and genetic characterization of the endoplasmic reticulum linoleoyl desaturase. J. Biol. Chem. 268:16345-16351.

Browse, J., Warwick, N., Somerville, C. R., and Slack, C. R. 1986. Fluxes through the prokaryotic and eukaryotic pathways of lipid synthesis in the '16:3' plant Arabidopsis thaliana. Biochem. J. 235:25-31.

Cecchini, N. M., Steffes, K., Schläppi, M. R., Gifford, A. N., and Greenberg, J. T. 2015. Arabidopsis AZI1 family proteins mediate signal mobilization for systemic defence priming. Nat. Commun. 6:7658.

Dyer, J. M., and Mullen, R. T. 2001. Immunocytological localization of two plant fatty acid desaturases in the endoplasmic reticulum. FEBS Lett. 494:44-47.

Eckardt, N. A. 2002. Plant disease susceptibility genes? Plant Cell 14:1983-1986.

Edwards, K., Johnstone, C., and Thompson, C. 1991. A simple and rapid method for the preparation of plant genomic DNA for PCR analysis. Nucleic Acids Res. 19:1349.

Engler, C., Kandzia, R., and Marillonnet, S. 2008. A one pot, one step, precision cloning method with high throughput capability. PLoS One 3:e3647.

Flattmann, K. 2015. Investigating the influence of dienoic fatty acids on aphid resistance in Arabidopsis. Undergraduate honors thesis, University of Arkansas, Fayetteville, AR, U.S.A.

Foster, S. P., Devine, G., and Devonshire, A. L. 2017. Insecticide Resistance. Pages 426-447 in:Aphids as Crop Pests, 2nd Ed., S. P. Foster, G. Devine, and A. L. Devonshire, eds. CAB International, Oxfordshire, U.K.

Froehlich, J. E., Wilkerson, C. G., Ray, W. K., McAndrew, R. S., Osteryoung, K. W., Gage, D. A., and Phinney, B. S. 2003. Proteomic study of the Arabidopsis thaliana chloroplastic envelope membrane utilizing alternatives to traditional two-dimensional electrophoresis. J. Proteome Res. 2:413-425.

Gibson, S., Arondel, V., Iba, K., and Somerville, C. 1994. Cloning of a temperature-regulated gene encoding a chloroplast $\omega-3$ desaturase from Arabidopsis thaliana. Plant Physiol. 106:1615-1621.

Goggin, F. L., Quisenberry, S. S., and Ni, X. 2017. Feeding Injury. Pages 303-322 in: Aphids as Crop Pests, 2nd Ed., H. F. van Emden, and R. Harrington, eds. CAB International, Oxfordshire, U.K.

Harrison, S. J., Mott, E. K., Parsley, K., Aspinall, S., Gray, J. C., and Cottage, A. 2006. A rapid and robust method of identifying transformed Arabidopsis thaliana seedlings following floral dip transformation. Plant Methods 2:19.

Harwood, L. 1980. Plant acyl lipids: Structure, distribution, and analysis. Page 1-55 in: The Biochemistry of Plants. P. K. Stumpf and E. E. Conn, eds. Vol. 4. Academic Press, New York.

He, Q., Naqvi, S., McLellan, H., Boevink, P. C., Champouret, N., Hein, I., and Birch, P. R. J. 2018. Plant pathogen effector utilizes host susceptibility factor NRL1 to degrade the immune regulator SWAP70. Proc. Natl. Acad. Sci. U.S.A. 115:E7834-E7843.

Heilmann, I., Mekhedov, S., King, B., Browse, J., and Shanklin, J. 2004. Identification of the Arabidopsis palmitoyl-monogalactosyldiacylglycerol $\triangle 7$-desaturase gene $F A D 5$, and effects of plastidial retargeting of Arabidopsis desaturases on the fad5 mutant phenotype. Plant Physiol. 136:4237-4245.

Hernández, M. L., Sicardo, M. D., and Martínez-Rivas, J. M. 2016. Differential contribution of endoplasmic reticulum and chloroplast $\omega-3$ fatty acid desaturase genes to the linolenic acid content of olive (Olea europaea) fruit. Plant Cell Physiol. 57:138-151.

Iwata, Y., Iida, T., Matsunami, T., Yamada, Y., Mishiba, K. I., Ogawa, T., Kurata, T., and Koizumi, N. 2018. Constitutive BiP protein accumulation in Arabidopsis mutants defective in a gene encoding chloroplast-resident stearoyl-acyl carrier protein desaturase. Genes Cells 23:456-465.

Jiang, C. J., Shimono, M., Maeda, S., Inoue, H., Mori, M., Hasegawa, M., Sugano, S., and Takatsuji, H. 2009. Suppression of the rice fattyacid desaturase gene OsSSI2 enhances resistance to blast and leaf blight diseases in rice. Mol. Plant-Microbe Interact. 22:820-829.

Kachroo, A., Venugopal, S. C., Lapchyk, L., Falcone, D., Hildebrand, D., and Kachroo, P. 2004. Oleic acid levels regulated by glycerolipid metabolism modulate defense gene expression in Arabidopsis. Proc. Natl. Acad. Sci. U.S.A. 101:5152-5157.

Kachroo, P., Kachroo, A., Lapchyk, L., Hildebrand, D., and Klessig, D. F. 2003. Restoration of defective cross talk in ssi2 mutants: Role of salicylic acid, jasmonic acid, and fatty acids in SSI2-mediated signaling. Mol. Plant-Microbe Interact. 16:1022-1029.
Kachroo, P., Shanklin, J., Shah, J., Whittle, E. J., and Klessig, D. F. 2001. A fatty acid desaturase modulates the activation of defense signaling pathways in plants. Proc. Natl. Acad. Sci. U.S.A. 98:9448-9453.

Kachroo, P., Venugopal, S. C., Navarre, D. A., Lapchyk, L., and Kachroo, A. 2005. Role of salicylic acid and fatty acid desaturation pathways in ssi2-mediated signaling. Plant Physiol. 139:1717-1735.

Kaloshian, I., and Walling, L. L. 2005. Hemipterans as plant pathogens. Annu. Rev. Phytopathol. 43:491-521.

Karki, N., Johnson, B. S., and Bates, P. D. 2019. Metabolically distinct pools of phosphatidylcholine are involved in trafficking of fatty acids out of and into the chloroplast for membrane production. Plant Cell 31:2768-2788.

Kasajima, I., Ide, Y., Ohkama-Ohtsu, N., Yoneyama, T., and Fujiwara, T. 2004. A protocol for rapid DNA extraction from Arabidopsis thaliana for PCR analysis. Plant Mol. Biol. Rep. 22:49-52.

Kennedy, S., Day, F., and Eastop, F. 1962. A Conspectus of Aphids as Vectors of Plant Viruses. Commonwealth Institute of Entomology, London.

Kuhar, T., Reiter, S., and Doughty, H. 2009. Green peach aphid on vegetables. VCE publication no. 2901-1081. Virginia Polytechnic Institute and State University, Petersburg, VA, U.S.A.

Kunst, L., Browse, J., and Somerville, C. 1989. A mutant of Arabidopsis deficient in desaturation of palmitic acid in leaf lipids. Plant Physiol. 90:943-947.

Lemieux, B., Miquel, M., Somerville, C., and Browse, J. 1990. Mutants of Arabidopsis with alterations in seed lipid fatty acid composition. Theor. Appl. Genet. 80:234-240.

Li, W., Deng, Y., Ning, Y., He, Z., and Wang, G. L. 2020. Exploiting broad-spectrum disease resistance in crops: From molecular dissection to breeding. Annu. Rev. Plant Biol. 71:575-603.

Li-Beisson, Y., Shorrosh, B., Beisson, F., Andersson, M. X., Arondel, V., Bates, P. D., Baud, S., Bird, D., Debono, A., Durrett, T. P., Franke, R. B., Graham, I. A., Katayama, K., Kelly, A. A., Larson, T., Markham, J. E., Miquel, M., Molina, I., Nishida, I., Rowland, O., Samuels, L., Schmid, K. M., Wada, H., Welti, R., Xu, C., Zallot, R., and Ohlrogge, J. 2013. Acyl-lipid metabolism. Arabidopsis Book 11:e0161.

Liu, L., and Li, J. 2019. Communications between the endoplasmic reticulum and other organelles during abiotic stress response in plants. Front. Plant Sci. 10:749.

Louis, J., Leung, Q., Pegadaraju, V., Reese, J., and Shah, J. 2010a. PAD4dependent antibiosis contributes to the ssi2-conferred hyper-resistance to the green peach aphid. Mol. Plant-Microbe Interact. 23:618-627.

Louis, J., Lorenc-Kukula, K., Singh, V., Reese, J., Jander, G., and Shah, J. 2010b. Antibiosis against the green peach aphid requires the Arabidopsis thaliana MYZUS PERSICAE-INDUCED LIPASE1 gene. Plant J. 64:800-811.

Louis, J., and Shah, J. 2013. Arabidopsis thaliana-Myzus persicae interaction: Shaping the understanding of plant defense against phloem-feeding aphids. Front. Plant Sci. 4:213.

Ma, X., Zhang, Q., Zhu, Q., Liu, W., Chen, Y., Qiu, R., Wang, B., Yang, Z., Li, H., Lin, Y., Xie, Y., Shen, R., Chen, S., Wang, Z., Chen, Y., Guo, J., Chen, L., Zhao, X., Dong, Z., and Liu, Y. G. 2015. A robust CRISPR/Cas9 system for convenient, high-efficiency multiplex genome editing in monocot and dicot plants. Mol. Plant 8:1274-1284.

Madey, E., Nowack, L. M., and Thompson, J. E. 2002. Isolation and characterization of lipid in phloem sap of canola. Planta 214:625-634.

Mandal, M. K., Chandra-Shekara, A. C., Jeong, R. D., Yu, K., Zhu, S., Chanda, B., Navarre, D., Kachroo, A., and Kachroo, P. 2012. Oleic acid-dependent modulation of NITRIC OXIDE ASSOCIATED1 protein levels regulates nitric oxide-mediated defense signaling in Arabidopsis. Plant Cell 24:1654-1674.

Martz, F., Kiviniemi, S., Palva, T. E., and Sutinen, M. L. 2006. Contribution of omega-3 fatty acid desaturase and 3-ketoacyl-ACP synthase II (KASII) genes in the modulation of glycerolipid fatty acid composition during cold acclimation in birch leaves. J. Exp. Bot. 57:897-909.

McConn, M., Creelman, R. A., Bell, E., Mullet, J. E., and Browse, J. 1997. Jasmonate is essential for insect defense in Arabidopsis. Proc. Natl. Acad. Sci. U.S.A. 94:5473-5477.

McConn, M., Hugly, S., Browse, J., and Somerville, C. 1994. A mutation at the fad8 locus of Arabidopsis identifies a second chloroplast $\omega-3 \mathrm{de}-$ saturase. Plant Physiol. 106:1609-1614.

Mehrshahi, P., Johnny, C., and DellaPenna, D. 2014. Redefining the metabolic continuity of chloroplasts and ER. Trends Plant Sci. 19:501-507.

Michaud, M., and Jouhet, J. 2019. Lipid trafficking at membrane contact sites during plant development and stress response. Front. Plant Sci. 10:2.

Minks, K., and Harrewijn, P. 1989. Aphids: Their Biology, Natural Enemies, and Control. Pages 61-89 in: World Crop Pests. Elsevier, Amsterdam.

Moloi, M. J., and van der Westhuizen, A. J. 2014. Involvement of nitric oxide in the Russian wheat aphid resistance response of wheat. Cereal Res. Commun. 42:119-125. 
Ning, Y., Liu, W., and Wang, G. L. 2017. Balancing immunity and yield in crop plants. Trends Plant Sci. 22:1069-1079.

Oppenheimer, D. G., Herman, P. L., Sivakumaran, S., Esch, J., and Marks, M. D. 1991. A myb gene required for leaf trichome differentiation in Arabidopsis is expressed in stipules. Cell 67:483-493.

Pegadaraju, V., Knepper, C., Reese, J., and Shah, J. 2005. Premature leaf senescence modulated by the Arabidopsis PHYTOALEXIN DEFICIENT4 gene is associated with defense against the phloem-feeding green peach aphid. Plant Physiol. 139:1927-1934.

Pegadaraju, V., Louis, J., Singh, V., Reese, J. C., Bautor, J., Feys, B. J., Cook, G., Parker, J. E., and Shah, J. 2007. Phloem-based resistance to green peach aphid is controlled by Arabidopsis PHYTOALEXIN DEFICIENT4 without its signaling partner ENHANCED DISEASE SUSCEPTIBILITY1. Plant J. 52:332-341.

Sato, Y., Shimizu-Inatsugi, R., Yamazaki, M., Shimizu, K. K., and Nagano, A. J. 2019. Plant trichomes and a single gene GLABRA1 contribute to insect community composition on field-grown Arabidopsis thaliana. BMC Plant Biol. 19:163.

Scala, A., Allmann, S., Mirabella, R., Haring, M. A., and Schuurink, R. C. 2013. Green leaf volatiles: A plant's multifunctional weapon against herbivores and pathogens. Int. J. Mol. Sci. 14:17781-17811.

Shah, J., Kachroo, P., Nandi, A., and Klessig, D. F. 2001. A recessive mutation in the Arabidopsis SSI2 gene confers SA- and NPRI-independent expression of $P R$ genes and resistance against bacterial and oomycete pathogens. Plant J. 25:563-574.

Shipley, M. M., Dillwith, J. W., Bowman, A. S., Essenberg, R. C., and Sauer, J. R. 1993. Changes in lipids of the salivary glands of the lone star tick, Amblyomma americanum, during feeding. J. Parasitol. 79:834-842.

Somerville, C., and Browse, J. 1996. Dissecting desaturation: Plants prove advantageous. Trends Cell Biol. 6:148-153.

Stevens, M., and Lacomme, C. 2017. Transmission of Plant Viruses. Pages 323-361 in: Aphids as Crop Pests, 2nd Ed., van Emden, H. F., and Harrington, R., eds. CAB International, Oxfordshire, U.K.

Upchurch, R. G. 2008. Fatty acid unsaturation, mobilization, and regulation in the response of plants to stress. Biotechnol. Lett. 30:967-977.

Van Emden, H. 2017. Host-Plant Resistance. Pages 515-531 in: Aphids as Crop Pests, 2nd Ed., H. F. van Emden and R. Harrington, eds. CAB International, Oxfordshire, U.K.

van Schie, C. C., and Takken, F. L. 2014. Susceptibility genes 101: How to be a good host. Annu. Rev. Phytopathol. 52:551-581.
Vaughn, K., Avila, A., Padilla, C., and Goggin, F. 2014. Development of fad7-1 single mutant Arabidopsis thaliana plants that are resistant to aphids. Discovery 15:94-99.

Vijayan, P., Shockey, J., Lévesque, C. A., Cook, R. J., and Browse, J. 1998. A role for jasmonate in pathogen defense of Arabidopsis. Proc. Natl. Acad. Sci. U.S.A. 95:7209-7214.

Walling, L. L. 2008. Avoiding effective defenses: Strategies employed by phloem-feeding insects. Plant Physiol. 146:859-866.

Wang, Z., and Benning, C. 2012. Chloroplast lipid synthesis and lipid trafficking through ER-plastid membrane contact sites. Biochem. Soc. Trans. 40:457-463.

Woźniak, A., Drzewiecka, K., Kęsy, J., Marczak, Ł., Narożna, D., Grobela, M., Motała, R., Bocianowski, J., and Morkunas, I. 2017. The influence of lead on generation of signaling molecules and accumulation of flavonoids in pea seedlings in response to pea aphid infestation. Molecules 22:1404.

Xia, Y., Yu, K., Navarre, D., Seebold, K., Kachroo, A., and Kachroo, P. 2010. The glabral mutation affects cuticle formation and plant responses to microbes. Plant Physiol. 154:833-846.

Yaeno, T., Matsuda, O., and Iba, K. 2004. Role of chloroplast trienoic fatty acids in plant disease defense responses. Plant J. 40:931-941.

Yara, A., Yaeno, T., Hasegawa, M., Seto, H., Montillet, J. L., Kusumi, K., Seo, S., and Iba, K. 2007. Disease resistance against Magnaporthe grisea is enhanced in transgenic rice with suppression of omega-3 fatty acid desaturases. Plant Cell Physiol. 48:1263-1274.

Yara, A., Yaeno, T., Montillet, J. L., Hasegawa, M., Seo, S., Kusumi, K., and Iba, K. 2008. Enhancement of disease resistance to Magnaporthe grisea in rice by accumulation of hydroxy linoleic acid. Biochem. Biophys. Res. Commun. 370:344-347.

Zaidi, S. S., Mukhtar, M. S., and Mansoor, S. 2018. Genome editing: Targeting susceptibility genes for plant disease resistance. Trends Biotechnol. 36:898-906.

Zhang, J., Liu, H., Sun, J., Li, B., Zhu, Q., Chen, S., and Zhang, H. 2012. Arabidopsis fatty acid desaturase FAD2 is required for salt tolerance during seed germination and early seedling growth. PLoS One 7:e30355.

Zhang, J. T., Zhu, J. Q., Zhu, Q., Liu, H., Gao, X. S., and Zhang, H. X. 2009. Fatty acid desaturase-6 (FAD6) is required for salt tolerance in Arabidopsis thaliana. Biochem. Biophys. Res. Commun. 390: 469-474.

Zuker, M. 2003. Mfold web server for nucleic acid folding and hybridization prediction. Nucleic Acids Res. 31:3406-3415. 